\title{
Multiplicative hyperring of fractions and coprime hyperideals
}

\author{
R. Ameri, A. Kordi and S. Hoskova-Mayerova
}

\begin{abstract}
In this paper we will introduce the notion of coprime hyperideals in multiplicative hyperrings and we will show some properties of them. Then we introduce the notion of hyperring of fractions generated by a multiplicative hyperring and then we will show some properties of them.
\end{abstract}

\section{Introduction}

The theory of hyperstructures was introduced by Marty in 1934 during the $8^{\text {th }}$ Congress of the Scandinavian Mathematicians [20]. Marty introduced hypergroups as a generalization of groups. He published some notes on hypergroups, using them in different contexts as algebraic functions, rational fractions, non commutative groups and since then, many researchers have worked on this new field of modern algebra and developed it. It was later observed that the theory of hyperstructures has many applications in both pure and applied sciences; for example, semi-hypergroups are the simplest algebraic hyperstructures that possess the properties of closure and associativity. The theory of hyperstructures has been widely reviewed $[6,7,8,10,13,21,25,28]$.

In [7] Corsini and Leoreanu-Fotea collected numerous applications of algebraic hyperstructures, especially those from the last fifteen years to the following subjects: geometry, hypergraphs, binary relations, lattices, fuzzy

Key Words: Multiplicative hyperring, Hyperideal, Prime, Coprime, Fundamental relation, Hyperring of fractions

2010 Mathematics Subject Classification: Primary 20N20; Secondary 16Y99.

Received: 01.12.2015

Revised: 01.02.2016.

Accepted: 04.04.2016 
sets and rough sets, automata, cryptography, codes, median algebras, relation algebras, artificial intelligence, and probabilities.

As mentioned e.g. in [9] the hyperrings have appeared as a new class of algebraic hyperstructures more general than that of hyperfields, introduced by Krasner [19] in the theory of valued fields. A Krasner hyperring is a nonempty set $\mathrm{R}$ endowed with a hyperoperation (the addition) and a binary operation (the multiplication) such that $(\mathrm{R} ;+)$ is a canonical hypergroup, $(\mathrm{R} ; \cdot)$ is a semigroup and the multiplication is distributive with respect to the addition. The theory of these hyperrings has been developing since the beginning of seventies, thanks to the contributions of Mittas [22, 23], Krasner [19], Stratigopoulos [39]. Some principal notions of hyperring theory can be found in [13, 14, 22, 36, 41]. Several types of hyperrings have been proposed e.g. in [24, 21, 42], in [40] the various types of hyperrings were given, with an outline of applications in chemistry and physics. The most general one were introduced by Spartalis [37], used also in the context of P-hyperrings or $(\mathrm{H} ; \mathrm{R})$-hyperrings [38]. A comprehensive review of hyperrings theory is covered in Nakassis [24] and in the book [14] written by Davvaz, Leoreanu-Fotea. The hyperrings were studied also by Ameri and Norouzi [1, 2, 3], Cristea and Jancic-Rasovic [9], Pelea [27] and others.

One important class of hyperrings was introduced by Rota in 1982, where the multiplication is a hyperoperation, while the addition is an operation, which is called multiplicative hyperrings (for more details see [32, 33, 34, 35]) and was subsequently investigated by Olson and Ward [26] and many others. De Salvo [15] introduced hyperrings in which the additions and the multiplications are hyperoperations. Moreover, there exist other types of hyperrings where both the addition and multiplication are hyperoperations and instead associativity, commutativity and distributivity satisfy weak associativity, weak commutativity and weak distributivity. This type of hyperrings, called $\mathrm{H}_{V^{-}}$ hyperrings, can be seen in $[40,41]$. Also, there are other types of hyperrings which were fully studied in [13]. These hyperrings are studied by Rahnamai Barghi [31]. Procesi and Rota in [29] have studied ring of fractions in Krasner hyperrings and also they conceptualized in [30] the notion of primeness of hyperideal in a multiplicative hyperring, and in [11], Dasgupta extended the prime and primary hyperideals in multiplicative hyperrings. Asokkumar and Velrajan $[4,5]$ have studied Von Neumann regularity in Krasner hyperrings.

Some equivalence relations - called fundamental relations - play important roles in the the theory of algebraic hyperstructures. The fundamental relations are one of the most important and interesting concepts in algebraic hyperstructures that ordinary algebraic structures are derived from algebraic hyperstructures by them. The fundamental relation $\beta^{*}$ on hypergroups was defined by Koskas [18], mainly studied by Corsini [20], Freni [16, 17], Vou- 
giouklis [41] (for more details about hyperrings and fundamental relations on hyperrings see $[1,2,12,13,36,41])$.

In this paper we consider the class of multiplicative hyperring as a hyperstructures $(R,+, \cdot)$, where $(R,+)$ is an abelian group, $(R,+)$ is a semihypergroup and the hyperoperation "." is distributive with respect to the operation " + ", i.e. $a \cdot(b+c) \subseteq a \cdot b+a \cdot c$. In this paper we introduce and study the notion of coprime (comaximal) hyperideals of multiplicative hyperrings and obtain their basic properties. Further, we introduce the notion of a hyperring of fractions of multiplicative hyperrings and investigate the basic properties such hyperrings.

\section{Preliminaries}

A hyperoperation "." on nonempty set $H$ is a mapping of $H \times H$ into the family of all nonempty subsets of $H$. Let "." be a hyperoperation on $H$. Then, $(H, \cdot)$ is called a hypergroupoid.

We can extend the hyperoperation on $H$ to subsets of $H$ as follows. For $A, B \subseteq H$ and $h \in H$, then

$$
A \cdot B=\cup_{a \in A, b \in B} a \cdot b, A \cdot h=A \cdot\{h\}, h \cdot B=\{h\} \cdot B .
$$

A semihypergroup is a hypergroupoid $(H, \cdot)$, which is associative, that is $(a$. $b) \cdot c=a \cdot(b \cdot c)$ for all $a, b, c \in H$. A hypergroup is a semihypergroup $(H, \cdot)$, that satisfies the reproduction axioms, that is $a \cdot H=H=H \cdot a$ for all $a \in H$.

A nonempty set $R$ with two hyperoperations "+" and "." is said to be a hyperring if $(R,+)$ is acanonical hypergroup, $(R, \cdot)$ is a semihypergroup with $r \cdot 0=0 \cdot r=0$ for all $r \in R(0$ as a bilaterally absorbing element) and the hyperoperation "." is distributive over + , i.e., for every $a, b, c \in R ; a \cdot(b+c)=$ $a \cdot b+a \cdot c$ and $(a+b) \cdot c=a \cdot c+b \cdot c$.

A multiplicative hyperring is an abelian group $(R,+)$ endowed with a hyperoperation ". " which satisfies the following conditions:

(R1) $\forall a, b, c \in R: a \cdot(b \cdot c)=(a \cdot b) \cdot c$;

(R2) $\forall a, b, c \in R:(a+b) \cdot c \subseteq a \cdot c+b \cdot c, a \cdot(b+c) \subseteq a \cdot b+a \cdot c$;

(R3) $\forall a, b \in R:(-a) \cdot b=a \cdot(-b)=-(a \cdot b)$.

If in $(R 2)$ the equality holds then we say that the multiplicative hyperring is strongly distributive.

Let $(R,+, \cdot)$ be a hyperring. We define the relation $\gamma$ as follows:

$a \gamma b$ if and only if $\{a, b\} \subseteq U$ where $U$ is a finite sum of finite products of elements of $R$, i.e.,

$$
a \gamma b \Leftrightarrow \exists z_{1}, \cdots, z_{n} \in R \text { such that }\{a, b\} \subseteq \sum_{j \in J} \prod_{i \in I_{j}} z_{i} ; \quad I_{j}, J \subseteq\{1, \cdots, n\} .
$$


We denote the transitive closure of $\gamma$ by $\gamma^{*}$. The relation $\gamma^{*}$ is the smallest equivalence relation on a multiplicative hyperring $(R,+, \cdot)$ such that the quotient $R / \gamma^{*}$, the set of all equivalence classes, is a fundamental ring. Let $\mathcal{U}$ be the set of all finite sums of products of elements of $R$ we can rewrite the definition of $\gamma^{*}$ on $R$ as follows:

$a \gamma^{*} b \Leftrightarrow \exists z_{1}, \ldots, z_{n+1} \in R$ with $z_{1}=a, z_{n+1}=b$ and $u_{1}, \ldots, u_{n} \in \mathcal{U}$ such that $\left\{z_{i}, z_{i+1}\right\} \subseteq u_{i}$ for $i \in\{1, \ldots, n\}$.

Suppose that $\gamma^{*}(a)$ is the equivalence class containing $a \in R$. Then, both the sum $\oplus$ and the product $\odot$ in $R / \gamma^{*}$ are defined as follows: $\gamma^{*}(a) \oplus \gamma^{*}(b)=$ $\gamma^{*}(c)$ for all $c \in \gamma^{*}(a)+\gamma^{*}(b)$ and $\gamma^{*}(a) \odot \gamma^{*}(b)=\gamma^{*}(d)$ for all $d \in \gamma^{*}(a) \cdot \gamma^{*}(b)$. Then $R / \gamma^{*}$ is a ring, which is called a fundamental ring of $R$ (see also [40]).

Definition 2.1. Let $R$ be a multiplicative hyperring. Then

(i) an element $e \in R$ is said to be a left (resp. right) identity if $a \in e \cdot a$ (resp. $a \in a \cdot e$ ) for $a \in R$. An element $e$ is called an identity element if it is both left and right identity element.

(ii) an element $e \in R$ is said to be a left (resp. right) scalar identity if $a=e \cdot a$ (resp., $a=a \cdot e$ ) for $a \in R$. An element $e$ is called an scalar identity element if it is both left and right scalar identity element.

(iii) Let $R$ be a multiplicative hyperring with an identity $e$. An element $A$ is called a left (right) invertible (with respect to $e$ ), if there exists $x \in R$, such that $e \in x \cdot a(e \in a \cdot x)$ and $a$ is called invertible if it is both a left and a right invertible.

A multiplicative hyperring $R$ is called a left (right) invertible if every element of $R$ has a left (right) invertible and $R$ is called an invertible if it is both a left and a right invertible. Denote the set of all invertible elements in $R$ by $U(R)$ (with respect to the identity $e$ by $U_{e}(R)$ ).

Definition 2.2. Let $R$ be a multiplicative hyperring. The element $e \in R$ is an idempotent if $0 \in e \cdot(1-e)$. Denote the set of all idempotent elements of $R$ by $\operatorname{Idem}(R)$.

Definition 2.3. Let $R$ be a multiplicative hyperring. Then we call $M_{n}(R)$ as the set of all hypermatixes of $R$. Also we called that for all $\mathcal{A}=\left(A_{i j}\right)_{n \times n}, \mathcal{B}$ $=\left(B_{i j}\right)_{n \times n} \in P^{*}\left(M_{n}(R)\right), \mathcal{A} \subseteq \mathcal{B}$ if and only if $A_{i j} \subseteq B_{i j}$.

Definition 2.4. We say that $I$ is a hyperideal of a multiplicative hyperring $(R,+,$.$) if it satisfies the following conditions:$

(i) $I-I \subseteq I$,

(ii) $\forall x \in I, r \in R, x \cdot r \cup r \cdot x \subseteq I$.

Definition 2.5. Let $R$ be a multiplicative hyperring. An element $a \in R$ is nilpotent, if there exists an integer $n$ such that $0 \in a^{n}$. Denote the set of all nilpotent elements of $R$ by $\operatorname{nil}(R)$. 
Definition 2.6. For an element $x$ of a multiplicative hyperring $R$, the left (right) annihilator of $x$ is $\operatorname{Ann}(x)=\{r \in R \mid r \cdot x=0\}(\operatorname{Ann}(x)=\{r \in$ $R \mid x \cdot r=0\}$ ). For a non-empty subset $B$ of a multiplicative hyperring $R$, the annihilator of $B$ is $A n n(B)=\cap\{A n n(x) \mid x \in B\}$.

Definition 2.7. [11] A hyperideal $P(\neq R)$ of a multiplicative hyperring $(R,+, \circ)$ is called a prime hyperideal of $R$, if for all $A, B \subseteq R, A \circ B \subseteq P$ then $A \subseteq P$ or $B \subseteq P$.

A hyperideal $I(\neq R)$ of a multiplicative hyperring $R$ is maximal in $R$ if for any hyperideal $J$ of $R, I \subsetneq J \subseteq R$ then $J=R$. Also, we say that $R$ is local, if it has just one maximal hyperideal.

Definition 2.8. [11] Let $\mathcal{C}$ be the class of all finite products of elements of a multiplicative hyperring $(R,+, \circ)$, i.e., $\mathrm{C}=\left\{\prod_{i=1}^{n} r_{i} \mid r_{i} \in R, i \in \mathbb{N}\right\} \subseteq P^{*}(R)$. A hyperideal $I$ of $R$ is said to be a $\mathcal{C}$-ideal of $R$ if, for any $A \in \mathcal{C}, A \cap I \neq \emptyset$, then $A \subseteq I$.

Definition 2.9. [14] A homomorphism (resp. good homomorphism) between two multiplicative hyperrings $(R,+, \circ)$ and $\left(R^{\prime},+^{\prime}, \circ^{\prime}\right)$ is a map $\phi$ from $R$ to $R^{\prime}$ such that for all $a, b \in R$,

$$
\begin{aligned}
& \phi(a+b)=\phi(a)+^{\prime} \phi(b) \quad \text { and } \quad \phi(a \circ b) \subseteq \phi(a) \circ^{\prime} \phi(b), \\
& \left(\text { resp. } \phi(a \circ b)=\phi(a) \circ^{\prime} \phi(b)\right) .
\end{aligned}
$$

\section{Coprime hyperideals in multiplicative hyperring}

Theorem 3.1. Let $R$ be a commutative multiplicative hyperring with scalar identity 1 . Then the hyperideal $I$ of $R$ is prime if and only if $I / \gamma^{*}$ be a prime ideal of $R / \gamma^{*}$.

Proof. $(\Rightarrow)$ Let for $x, y \in R / \gamma^{*}, x \cdot y \in I / \gamma^{*}$. Thus, there exist $a, b \in R$ such that $x=\gamma^{*}(a), y=\gamma^{*}(b)$ and $x y=\gamma^{*}(a) \gamma^{*}(b)=\gamma^{*}(a \cdot b)$. So, $\gamma^{*}(a) \gamma^{*}(b)=$ $\gamma^{*}(a \cdot b) \in I / \gamma^{*}$, then $a \cdot b \subseteq I$. Since $I$ is prime, then $a \in I$ or $b \in I$. Hence $x=\gamma^{*}(a) \in I / \gamma^{*}$ or $y=\gamma^{*}(b) \in I$.

$(\Leftarrow)$ Assume that $a \cdot b \subseteq I$ for $a, b \in R$, then $\gamma^{*}(a), \gamma^{*}(b) \in R / \gamma^{*}$ and $\gamma^{*}(a) \gamma^{*}(b)=\gamma^{*}(a \cdot b) \in I / \gamma^{*}$. Therefore $\gamma^{*}(a) \in I / \gamma^{*}$ or $\gamma^{*}(b) \in I / \gamma^{*}$. Hence $a \in I$ or $b \in I$, i.e., $I$ is a prime hyperideal of $R$.

Theorem 3.2. Let $R$ be a commutative multiplicative hyperring with scalar identity 1 and $I$ be a left hyperideal of $R$. If $M_{n}(I)$ is a prime hyperideal of $M_{n}(R)$, then $I$ is prime hyperideal of $R$. 
Proof. Assume that $a, b \in R$ such that $a b \subseteq I$. Then

$$
\left(\begin{array}{cccc}
a b & 0 & \ldots & 0 \\
0 & 0 & \ldots & 0 \\
\vdots & \vdots & \ddots & \vdots \\
0 & 0 & \ldots & 0
\end{array}\right) \subseteq M_{n}(I)
$$

But

$$
\left(\begin{array}{cccc}
a b & 0 & \ldots & 0 \\
0 & 0 & \ldots & 0 \\
\vdots & \vdots & \ddots & \vdots \\
0 & 0 & \ldots & 0
\end{array}\right)=\left(\begin{array}{cccc}
a & 0 & \ldots & 0 \\
0 & 0 & \ldots & 0 \\
\vdots & \vdots & \ddots & \vdots \\
0 & 0 & \ldots & 0
\end{array}\right)\left(\begin{array}{cccc}
b & 0 & \ldots & 0 \\
0 & 0 & \ldots & 0 \\
\vdots & \vdots & \ddots & \vdots \\
0 & 0 & \ldots & 0
\end{array}\right)
$$

Therefore

$$
\left(\begin{array}{cccc}
a & 0 & \ldots & 0 \\
0 & 0 & \ldots & 0 \\
\vdots & \vdots & \ddots & \vdots \\
0 & 0 & \ldots & 0
\end{array}\right) \in M_{n}(I) \quad \text { or } \quad\left(\begin{array}{cccc}
b & 0 & \ldots & 0 \\
0 & 0 & \ldots & 0 \\
\vdots & \vdots & \ddots & \vdots \\
0 & 0 & \ldots & 0
\end{array}\right) \in M_{n}(I) .
$$

Hence $a \in I$ or $b \in I$, i.e., $I$ is a prime hyperideal of $R$.

Proposition 3.3. Every non zero commutative multiplicative hyperring $R$ with scalar identity 1 , has at least one maximal hyperideal.

Proof. Let $\Sigma$ be the set of all hyperideals $\neq<1>$. Since $0 \in \Sigma$, then $\Sigma$ is not empty. Assume that $\left\langle I_{t}\right\rangle$ be a chain of hyperideals in $\Sigma$, so that for each pair of indices $t_{1}, t_{2}$ we have either $I_{t_{1}} \subseteq I_{t_{2}}$ or $I_{t_{2}} \subseteq I_{t_{1}}$. Let $I=\bigcup_{t} I_{t}$. It is clear that $I$ is a hyperideal. Since for all $t, 1 \notin I_{t}$ we have $1 \notin I$. Hence $I \in \Sigma$ and $I$ is an upper bound of the chain. Therefore by Zorn's lemma $\Sigma$ has a maximal element.

Theorem 3.4. Let $R$ be a commutative multiplicative hyperring with scalar identity 1 . Then we have the following statements:

(i) Let $M$ be a proper hyperideal of $R$ such that every $x \in R \backslash M$ is invertible in $R$. Then $R$ is a local multiplicative hyperring and $M$ is the only maximal hyperideal of $R$.

(ii) Let $M$ be a maximal hyperideal of $R$ such that every element of $1+M$ be invertible in $R$ and also for all $x \notin M,\langle x\rangle=R \cdot x$. Then $R$ is a local multiplicative hyperring.

Proof. (i) Let $I$ be a proper hyperideal of $R$. Then $I$ has not any invertible elements. Hence $M$ is the only maximal hyperideal of $R$, i.e., $R$ is local 
multiplicative hyperring.

(ii) Let $x \in R \backslash M$. Since $M$ is maximal hyperideal then $\langle x, M\rangle=R$. So, there exist $m \in M$ such that $1 \in\langle x\rangle+m$. Thus $1-m \in\langle x\rangle$. Since $1-m \in 1+M$, then it's invertible. Therefore there exists $\ell \in R$ such that $1 \in(1-m) \cdot \ell$, so, $1 \in\langle x\rangle \cdot \ell, x$ is invertible. Hence by $(i), R$ is local multiplicative hyperring.

Theorem 3.5. The set $\Upsilon$ of all nilpotent elements in a commutative multiplicative hyperring $R$ with scalar identity 1 , is a hyperideal, and $R / \Upsilon$ has no nonzero nilpotent element.

Proof. Assume that $x \in \Upsilon$, there exists $n \in \mathbb{N}$ such that $0 \in x^{n}$. Thus for all $r \in R$, we have $0 \in r \cdot x^{n}=(r \cdot x)^{n}$, so $r \cdot x \subseteq \Upsilon$ and similarly $x \cdot r \subseteq \Upsilon$. Hence $r \cdot x \cup x \cdot r \subseteq \Upsilon$. Now, assume that $x, y \in \Upsilon$, then there exist $n, m \in \mathbb{N}$ such that $0 \in x^{n}$ and $0 \in y^{m}$. Therefore, we have $0 \in(x-y)^{n+m}$. Hence $x-y \in \Upsilon$, i.e., $\Upsilon-\Upsilon \subseteq \Upsilon$ and therefore $\Upsilon$ is a hyperideal.

Now, assume that $x+\Upsilon \in R / \Upsilon$, where $x \in R$. Let $0_{R / \Upsilon} \in(x+\Upsilon)^{n}$ for some $n \in \mathbb{N}$ then $x^{n} \in \Upsilon$, so there exists $k \in \mathbb{N}$ such that $0 \in\left(x^{n}\right)^{k}=x^{n k}$. Therefore $x \in \Upsilon$, i.e., $x+\Upsilon=\Upsilon=0_{R / \Upsilon}$.

Theorem 3.6. Let $R$ be a commutative multiplicative hyperring with scalar identity 1 , which every element $x \in R, x \in x^{n}$ for some $n \geq 2$. Then every prime $\mathcal{C}$-ideal in $R$ is maximal hyperideal.

Proof. Let $P$ be a prime hyperideal of $R$. For all non zero element $x$ in $R-P$ there exists $n \geq 2$ such that $x \in x^{n}$. Since $0 \in\left(x-x^{n}\right) \cap P$, we have $\left(x-x^{n}\right) \subseteq P$, also $x\left(1-x^{n-1}\right) \subseteq x-x^{n}$. So since $x \notin P, 1-x^{n-1} \subseteq P$. It's clear that $1 \in x^{n-1}$, therefore $x$ is invertible. Hence by Theorem 3.4 $(i), P$ is a maximal hyperideal of $R$.

Theorem 3.7. Let $R$ be a commutative multiplicative hyperring with scalar identity 1. Let $I_{1}, I_{2}, \cdots, I_{n}$ be hyperideals and $P$ be a prime hyperideal containing $\cap_{i=1}^{n} I_{i}$. Then $I_{i} \subseteq P$ for some $i$. If $P=\cap_{i=1}^{n} I_{i}$, then for some $i$, $P=I_{i}$.

Proof. Suppose that $I_{i} \nsubseteq P$ for all $1 \leq i \leq n$, then there exists $x_{i} \in I_{i}$ such that $x_{i} \notin P$. Thus $\prod x_{i} \subseteq \prod I_{i} \subseteq \cap I_{i}$, but since $P$ is prime hyperideal $\prod x_{i} \nsubseteq P$. Hence $\cap I_{i} \nsubseteq P$.

Now, if $P=\cap_{i=1}^{n} I_{i}$, then $P \subseteq I_{i}$. Therefore $P=I_{i}$ for some $1 \leq i \leq n$.

Let $I, J$ be two hyperideals in a commutative multiplicative hyperring. Then define:

$$
(I: J)=\{x \in R \mid x \cdot J \subseteq I\}
$$


Theorem 3.8. Let $R$ be a multiplicative hyperring and $I, J$ are two hyperideals in $R$. Then $(I: J)$ is a hyperideal of $R$.

Proof. For all $x, y \in(I: J)$, we have $x \cdot J \subseteq I, y \cdot J \subseteq I$, then $(x-y) \cdot J \subseteq$ $x \cdot J-y \cdot J \subseteq I-I \subseteq I$. Also, for all $x \in(I: J)$ and $r \in R$ we have $r \cdot x \cdot J \subseteq r \cdot I \subseteq I$,i.e., $r \cdot x \subseteq(I: J)$ and similarly $x \cdot r \subseteq(I: J)$. Hence $(I: J)$ is a hyperideal of $R$.

Theorem 3.9. Let $R$ be a commutative multiplicative hyperring and $I, J$ and $K$ be hyperideals in $R$. Then we have the following statements:

(i) $I \subseteq(I: J)$,

(ii) $(I: J) \cdot J \subseteq I$,

(iii) $((I: J): K)=(I: J \cdot K)=((I: K): J)$,

(iv) $\left(\cap_{i} I_{i}: J\right)=\cap_{i}\left(I_{i}: J\right)$,

(v) $\cap_{i}\left(I: J_{i}\right)=\left(I: \sum_{i} J_{i}\right)$.

Proof. It's straightforward.

Theorem 3.10. Let $R$ be a commutative multiplicative hyperring and $I, J$ are hyperideals in $R$. If $I$ is prime hyperideal, then $(I: J)=I$ or $R$.

Proof. Since $I$ is prime hyperideal, then we have $(I: J)=\{x \in R \mid x \cdot J \subseteq I\}=$ $\{x \in R \mid x \in I$ or $J \subseteq I\}=I$ or $R$.

Theorem 3.11. Let $I, J$ be hyperideals of commutative multiplicative hyperring $R$ with scalar identity 1 and $I \subseteq J$. If

(i) $J / I$ is cyclic,

(ii) $J$ and $J \cdot(I: J)$ are idempotent,

(iii) $I$ and $J \cdot(I: J)$ are $\mathcal{C}$-ideal,

then we have $I=J \cdot(I: J)$.

Proof. Assume that $J=I+\langle a\rangle$ for $a \in R$. Let $K=J \cdot(I: J)$, then $K \subseteq I$. Thus $K \subseteq K^{2} \subseteq I^{2} \subseteq J \cdot I \subseteq J \cdot(I: J)=K$. So $I^{2}=K$. Now, let $x \in I$. Then, since $I \subseteq J \subseteq J^{2}$, we have $x \in y+a \cdot r+n a$ where $y \in I^{2}, r \in R$ and $n \in \mathbb{Z}$. Therefore, there exists $t \in a \cdot r+n a$ such that $x-y=t$, i.e., $a \cdot r+n a \cap I \neq \emptyset$ and since $I$ is $\mathcal{C}$-ideal, then $a \cdot\left(r+n \cdot 1_{R}\right) \subseteq a \cdot r+n a \subseteq I$. Since $r+n \cdot 1_{R} \in(I: a)=(I: J)$, we have $a \cdot\left(r+n \cdot 1_{R}\right) \subseteq J \cdot(I: J)=K$. Thus $(a \cdot r+n a) \cap K \neq \emptyset$. Since $K$ is $\mathcal{C}$-ideal, then $a \cdot r+n a \subseteq K$. Hence $x \in y+a \cdot r+n a \subseteq I^{2}+K \subseteq K$.

Definition 3.12. Let $R$ be a commutative multiplicative hyperring and $I, J$ be hyperideals in $R$ with scalar identity 1 . We said that $I, J$ are coprime (comaximal) if $I+J=R$. 
Example 3.13. Let $R=\mathbb{Z} \bigoplus \mathbb{Z}$ and define

$$
(a, b) \circ(c, d)=\left\{\begin{array}{ll}
(a c, \mathbb{Z}) ; \quad b d \neq 0 \\
(a c, 0) ; \quad b d=0
\end{array} .\right.
$$

Then $(R,+, \circ)$ is a multiplicative hyperring such that is not strongly distributive, because by considering $a=(1,1), b=(0,2), c=(0,-2)$, we have $a \circ(b+c)=(1,1) \circ(0,0)=(0,0)$ but $a \circ b+a \circ c=(0, \mathbb{Z})+(0, \mathbb{Z})=(0, \mathbb{Z}+\mathbb{Z})$. Now, let $I=(\mathbb{Z}, 0)$ be a strongly distributive hyperideal of $R$ and $e=(1,0)$ is scalar identity element of $I$. It is clear that $\operatorname{Ann}(I)=(0, \mathbb{Z})$ and $R=$ $I+\operatorname{Ann}(I)$. Therefore, $I$ and $A n n(I)$ are coprime.

Theorem 3.14. Let $R$ be a commutative multiplicative hyperring and $I, J$ are hyperideals in $R$ with scalar identity 1 . Then we have the following statements:

(i) If $I, J$ are coprime, then $I^{n}, J^{m}$ are also coprime for all $m, n \in \mathbb{N}$.

(ii) If $I, J$ are coprime, then $\operatorname{rad}(I), \operatorname{rad}(J)$ are coprime.

(iii) If $I, J$ are coprime, then $(I: J)=I$.

(iv) $I, J$ are coprime if and only if $M_{n}(I), M_{n}(J)$ are coprime.

Proof. ( $i$ ) Assume that $I^{n}+J^{m} \neq R$. Then there exists a prime hyperideal $P$ such that $I^{n}+J^{m} \subseteq P$. Thus $I^{n} \subseteq P$ and $J^{m} \subseteq P$. Since $P$ is a prime hyperideal then we have $I, J \subseteq P$. But $I+J \subseteq P$ and it is a contradiction.

(ii) Since $R=\operatorname{rad}(R)=\operatorname{rad}(I+J) \subseteq \operatorname{rad}(\operatorname{rad}(I)+\operatorname{rad}(J))$, then $\operatorname{rad}(\operatorname{rad}(I)+\operatorname{rad}(J))=R$, i.e., $\operatorname{rad}(I)+\operatorname{rad}(J)=R$.

(iii) By Theorem 3.9(v) and since $I, J$ are coprime, we have $(I: J)=$ $(I: I+J)=(I: R)=I$.

(iv) Let $I, J$ are coprime, then $I+J=R$. Thus $M_{n}(I)+M_{n}(J)=$ $M_{n}(I+J)=M_{n}(R)$, i.e., $M_{n}(I), M_{n}(J)$ are coprime.

The converse is straightforward.

Let $f: R \rightarrow R^{\prime}$ be a good homomorphism from multiplicative hyperring $R$ to $R^{\prime}$. We define the extension $I^{e}$ of hyperideal $I$ to be the generated hyperideal by $f(I)$ in $R^{\prime}$. Now, if $J$ is a hyperideal of $R^{\prime}$, then $f^{-1}(J)$ is always an hyperideal of $R$, called the contraction $J^{c}$ of $J$.

Theorem 3.15. Let $f: R \rightarrow R^{\prime}$ be a good homomorphism from multiplicative hyperring $R$ to $R^{\prime}$. Then we have the following statements:

(i) If $I$ is a prime hyperideal of $R^{\prime}$, then $I^{c}$ is prime hyperideal of $R$.

(ii) If $I^{c}, J^{c}$ are coprime in $R^{\prime}$ and $f$ is onto, then $I, J$ are coprime in $R$.

Proof. (i) Assume that $I$ is a hyperideal of $R^{\prime}$. Let $a, b \in R$ such that $a \cdot b \subseteq$ $f^{-1}(I)$. Then $f(a \cdot b) \subseteq I$ and so $f(a) \in I$ or $f(b) \in I$. Hence $a \in f^{-1}(I)$ or $b \in f^{-1}(I)$, i.e., $f^{-1}(I)$ is prime hyperideal of $R$. 
(ii) Suppose that $I^{c}, J^{c}$ are coprime in $R^{\prime}$, then $I^{c}+J^{c}=R^{\prime}$. Since $I^{c}+J^{c} \subseteq$ $(I+J)^{c}$, thus $(I+J)^{c}=R^{\prime}$. Therefore $I+J=R$, by $f$ is onto.

Theorem 3.16. (Chinese Remainder Theorem) Suppose that $I_{1}, \cdots, I_{n}$ are coprime hyperideals of multiplicative hyperring $R$ with scalar identity 1 . Then the canonical good homomorphism $\phi: R \rightarrow \prod_{i=1}^{n} R / I_{i}$ is onto.

Proof. It's enough to show that $\left(0, \ldots, r+I_{i}, 0, \ldots, 0\right) \in \operatorname{Im}(\phi)$ for all $1 \leq i \leq$ $n$. We need to show that $(1,0, \ldots, 0) \in \operatorname{Im}(\phi)$, i.e., there exists $r \in R$ such that $1-r \in I_{1}$ and $r \in I_{2} \cap \cdots \cap I_{n}$. For all $j>1$, since $I_{1}, I_{j}$ are comaximal, then there exist $a_{1 j} \in I_{1}$ and $a_{j} \in I_{j}$ such that $1=a_{1 j}+a_{j}$. Thus we have, $1 \in\left(a_{12}+a_{2}\right)\left(a_{13}+a_{3}\right) \cdots\left(a_{1 n}+a_{n}\right) \subseteq A+a_{2} \cdots a_{n}$, where $A$ is a sum of terms from $I_{1}$. Therefore, letting $r \in a_{2} \ldots a_{n}$ we have $1-r \in A \subseteq I_{1}$ and $r \in I_{2} \cap \ldots \cap I_{n}$, as desired.

Corollary 3.17. If $I_{1}, \ldots, I_{n}$ are comprime hyperideals of multiplicative hyperring $R$ with scalar identity 1 , then $R / \bigcap_{i=1}^{n} I_{i} \cong \prod_{i=1}^{n} R / I_{i}$.

Proof. Assume that $\phi(r)=(0, \ldots, 0)$. Then for all $i$, we have $r+I_{i}=0_{R / I_{i}}$. Therefore $r \in I_{i}$ for all $i$, i.e., $\operatorname{ker}(\phi)=\bigcap_{i=1}^{n} I_{i}$. Hence by Theorem 3.16, $R / \bigcap_{i=1}^{n} I_{i} \cong \prod_{i=1}^{n} R / I_{i}$.

Theorem 3.18. Let $R$ be a multiplicative hyperring with scalar identity 1 and for all $r \in R$ and $e \in \operatorname{Idem}(R),|r \cdot e|=1$. Then the following statements are equivalent:

(i) $R \cong R_{1} \times R_{2}$, where neither of the hyperrings $R_{1}$ and $R_{2}$ is the zero hyperring,

(ii) $R$ contains the non trivial idempotents.

Proof. $(i) \Rightarrow($ ii $)$ Let $e$ be a non trivial idempotent in $R$. Define the good homomorphism $\phi: R \rightarrow R /<e>\times R /<1-e>$ by $\phi(r)=(r+<e>$, $r+<1-e>$ ). It's clear that $\langle e>,<1-e>$ are coprime, then by Chinese Remainder Theorem $\phi$ is epimorphism and for all $r \in \operatorname{ker}(\phi)$ we have $(r+<e>, r+<1-e>)=\left(0_{R /<e>}, 0_{R /<1-e>}\right)$, i.e., $r \in<e>\cap<1-e>$ and since for all $\ell \in R,|\ell \cdot e|=1$, then $r=0$, therefore $\operatorname{ker}(\phi)=\{0\}$. Now by Corollary 3.17, we have $R \cong R /\langle e\rangle \times R /<1-e\rangle$.

$($ ii $) \Rightarrow(i)$ Assume that $e$ be a non trivial idempotent in $R$. Since $R \cong R /<$ $e>\times R /<1-e>$ then $(1,0) \in R /<e>\times R /<1-e>$ is a non trivial idempotent. 


\section{Multiplicative hyperring of fractions}

Definition 4.1. A nonempty subset $S$ of multiplicative hyperring $(R,+, \cdot)$ with scalar identity 1 is called a multiplicative closed subset (MCS), if it has the following properties:

(i) $1 \in S$, (ii) $a \cdot S=S \cdot a=S$ for all $a \in S$.

Let $S$ be a MCS of $(R,+, \cdot)$. We shall construct the hyperring of fractions $S^{-1} R$. Let us consider the following relation in $R \times S$ :

$$
(r, s) \sim\left(r^{\prime}, s^{\prime}\right) \Longleftrightarrow \gamma^{*}\left(A \cdot r \cdot s^{\prime}\right)=\gamma^{*}\left(A \cdot r^{\prime} \cdot s\right),
$$

for some nonempty subset $A$ of $S$.

Theorem 4.2. $\sim$ is an equivalence relation on $R \times S$.

Proof. It's clear that $\sim$ is reflexive and symmetric. Now, assume that $\left(r_{1}, s_{1}\right) \sim$ $\left(r_{2}, s_{2}\right)$ and $\left(r_{2}, s_{2}\right) \sim\left(r_{3}, s_{3}\right)$. Then there exist $A, B \subseteq S$ such that $\gamma^{*}(A$. $\left.r_{1} \cdot s_{2}\right)=\gamma^{*}\left(A \cdot r_{2} \cdot s_{1}\right)$ and $\gamma^{*}\left(B \cdot r_{2} \cdot s_{3}\right)=\gamma^{*}\left(B \cdot r_{3} \cdot s_{2}\right)$. Thus we have $\gamma^{*}\left(A \cdot B \cdot r_{2} \cdot s_{3} \cdot s_{1}\right)=\gamma^{*}\left(A \cdot B \cdot r_{3} \cdot s_{2} \cdot s_{1}\right)$, which implies that $\gamma^{*}\left(B \cdot\left(A \cdot r_{2} \cdot s_{1}\right) \cdot s_{3}\right)=$ $\gamma^{*}\left(A \cdot B \cdot r_{3} \cdot s_{2} \cdot s_{1}\right)$, and hence $\gamma^{*}\left(B \cdot\left(A \cdot r_{1} \cdot s_{2}\right) \cdot s_{3}\right)=\gamma^{*}\left(A \cdot B \cdot r_{3} \cdot s_{2} \cdot s_{1}\right)$. So that $\gamma^{*}\left(A \cdot B \cdot s_{2}\right) \gamma^{*}\left(r_{1} \cdot s_{3}\right)=\gamma^{*}\left(A \cdot B \cdot s_{2}\right) \gamma^{*}\left(r_{3} \cdot s_{1}\right)$. Therefore $\sim$ is transitive.

Now we define the following hyperoperations on $S^{-1} R$,

$\left(r_{1}, s_{1}\right) \oplus\left(r_{2}, s_{2}\right)=\left(r_{1} \cdot s_{2}+r_{2} \cdot s_{1}, s_{1} \cdot s_{2}\right)$

and

$$
=\left\{(r, s) \mid r \in r_{1} \cdot s_{2}+r_{2} \cdot s_{1}, s \in s_{1} \cdot s_{2}\right\}
$$

$$
\left(r_{1}, s_{1}\right) \odot\left(r_{2}, s_{2}\right)=\left(r_{1} \cdot r_{2}, s_{1} \cdot s_{2}\right)=\left\{(r, s) \mid r \in r_{1} \cdot r_{2}, s \in s_{1} \cdot s_{2}\right\} .
$$

We need to show that $\oplus$ and $\odot$ are well defined. If $\left(r_{1}, s_{1}\right)=\left(a_{1}, t_{1}\right)$ and $\left(r_{2}, s_{2}\right)=\left(a_{2}, t_{2}\right)$, then there exist $A, B \subseteq S$ such that

$$
\begin{aligned}
& \gamma^{*}\left(A \cdot r_{1} \cdot t_{1}\right)=\gamma^{*}\left(A \cdot a_{1} \cdot s_{1}\right) \\
& \gamma^{*}\left(B \cdot r_{2} \cdot t_{2}\right)=\gamma^{*}\left(B \cdot a_{2} \cdot s_{2}\right)
\end{aligned}
$$

Multiplying (1) by $\gamma^{*}\left(B \cdot t_{2} \cdot s_{2}\right)$ and (2) by $\gamma^{*}\left(A \cdot t_{1} \cdot s_{1}\right)$. Then we have $\gamma^{*}\left(A \cdot B \cdot s_{1} \cdot s_{2} \cdot t_{2} \cdot a_{1}\right)=\gamma^{*}\left(A \cdot B \cdot s_{2} \cdot t_{2} \cdot t_{1} \cdot r_{1}\right)$ and $\gamma^{*}\left(A \cdot B \cdot s_{1} \cdot s_{2} \cdot\right.$ $\left.t_{1} \cdot a_{2}\right)=\gamma^{*}\left(A \cdot B \cdot s_{1} \cdot t_{1} \cdot t_{2} \cdot r_{2}\right)$. Now, by adding these equalities we obtain $\gamma^{*}\left(A \cdot B \cdot\left(s_{1} \cdot s_{2} \cdot\left(t_{2} \cdot a_{1}+t_{1} \cdot a_{2}\right)\right)\right)=\gamma^{*}\left(A \cdot B \cdot\left(t_{1} \cdot t_{2} \cdot\left(r_{1} \cdot s_{2}+r_{2} \cdot s_{1}\right)\right)\right)$. Hence $\left(r_{1} \cdot s_{2}+r_{2} \cdot s_{1}, s_{1} \cdot s_{2}\right)=\left(a_{1} \cdot t_{2}+a_{2} \cdot t_{1}, t_{1} \cdot t_{2}\right)$, i.e., $\oplus$ is well defined. Again, multiplying (1) by (2) we obtain $\gamma^{*}\left(A \cdot B \cdot\left(r_{1} \cdot r_{2}\right) \cdot\left(t_{1} \cdot t_{2}\right)\right)=$ $\gamma^{*}\left(A \cdot B \cdot\left(a_{1} \cdot a_{2}\right) \cdot\left(s_{1} \cdot s_{2}\right)\right)$. Therefore $\left(r_{1} \cdot r_{2}, s_{1} \cdot s_{2}\right)=\left(a_{1} \cdot a_{2}, t_{1} \cdot t_{2}\right)$, i.e., $\odot$ is well defined. 
Theorem 4.3. Let $(R,+, \cdot)$ be a commutative multiplicative hyperring with scalar identity 1 and $S$ be a $M C S$ of $R$. Then $\left(S^{-1} R, \oplus, \odot\right)$ is a commutative hyperring with scalar identity.

Proof. By above discussion $\oplus$ and $\odot$ are well defined. Now we need to show that $\left(S^{-1} R, \oplus\right)$ is a hypergroup and $\left(S^{-1} R, \odot\right)$ is an associative hyperoperation, which is distributive with respect to $\oplus$. If $\left(r_{1}, s_{1}\right),\left(r_{2}, s_{2}\right),\left(r_{3}, s_{3}\right) \in$ $S^{-1} R$, then we have:

$$
\begin{aligned}
\left(r_{1}, s_{1}\right) \oplus\left[\left(r_{2}, s_{2}\right)\right. & \left.\oplus\left(r_{3}, s_{3}\right)\right]=\left(r_{1}, s_{1}\right) \oplus\left(r_{2} \cdot s_{3}+r_{3} \cdot s_{2}, s_{2} \cdot s_{3}\right) \\
& =\left(r_{1} \cdot\left(s_{2} \cdot s_{3}\right)+\left(r_{2} \cdot s_{3}+r_{3} \cdot s_{2}\right) \cdot s_{1}, s_{1} \cdot s_{2} \cdot s_{3}\right) \\
& =\left(r_{1} \cdot\left(s_{2} \cdot s_{3}\right)+r_{2} \cdot s_{1} \cdot s_{3}+r_{3} \cdot s_{1} \cdot s_{2}, s_{1} \cdot s_{2} \cdot s_{3}\right) \\
& =\left[\left(r_{1}, s_{1}\right) \oplus\left(r_{2}, s_{2}\right)\right] \oplus\left(r_{3}, s_{3}\right) .
\end{aligned}
$$

It means that $\left(S^{-1} R, \oplus\right)$ is associative. Now, we prove the reproduction axioms for $\left(S^{-1} R, \oplus\right)$. For every $\left(r_{1}, s_{1}\right),\left(r_{2}, s_{2}\right) \in S^{-1} R$ we have $s_{1}, s_{2} \in S$ and by definition there exists $s_{3} \in S$ such that $s_{1} \in s_{2} \cdot s_{3}$. Since $(R,+)$ is a group then reproduction axioms hold for the additive law in $R$, we have $r_{2} \cdot s_{3}+\left(s_{2}+1\right) \cdot R=R$. Therefore, there exists $r_{3} \in R$ such that $r_{1} \in$ $r_{2} \cdot s_{3}+s_{2} \cdot r_{3}+r_{3}$ which implies that $r_{1} \in r_{2} \cdot s_{3}+r_{3} \cdot s_{2}+r_{3} \cdot 1 \subseteq r_{2} \cdot s_{3}+$ $r_{3} \cdot s_{2}+r_{3} \cdot s_{4} \cdot s_{2}$, where $1 \in s_{2} \cdot s_{4}$. Then $r_{1} \in r_{2} \cdot s_{3}+\left(r_{3}+r_{3} \cdot s_{4}\right) \cdot s_{2}$. Therefore, there exists $a \in r_{3}+r_{3} \cdot s_{4}$ such that $r_{1} \in r_{2} \cdot s_{3}+a \cdot s_{2}$. Hence $\left(r_{1}, s_{1}\right) \in\left(r_{2}, s_{2}\right) \oplus\left(a, s_{3}\right)$, which implies that $S^{-1} R \subseteq\left(r_{2}, s_{2}\right) \oplus S^{-1} R$, so $S^{-1} R=\left(r_{2}, s_{2}\right) \oplus S^{-1} R$. Also, $\odot$ is distributive with respect to $\oplus$, because for all $\left(r_{1}, s_{1}\right),\left(r_{2}, s_{2}\right),\left(r_{3}, s_{3}\right) \in S^{-1} R$, then we have:

$$
\begin{aligned}
\left(r_{1}, s_{1}\right) \odot\left[\left(r_{2}, s_{2}\right) \oplus\left(r_{3}, s_{3}\right)\right] & =\left(r_{1}, s_{1}\right) \odot\left(r_{2} \cdot s_{3}+r_{2} \cdot s_{2}, s_{2} \cdot s_{3}\right) \\
& =\left(r_{1} \cdot\left(r_{2} \cdot s_{3}+r_{2} \cdot s_{2}\right), s_{1} \cdot s_{2} \cdot s_{3}\right) \\
& =\left(r_{1} \cdot r_{2} \cdot s_{3}+r_{1} \cdot r_{2} \cdot s_{2}, s_{1} \cdot s_{2} \cdot s_{3}\right) \\
& =\left(r_{1} \cdot r_{2} \cdot s_{3} \cdot s_{1}+r_{1} \cdot r_{2} \cdot s_{2} \cdot s_{1}, s_{1} \cdot s_{2} \cdot s_{3} \cdot s_{1}\right) \\
& =\left[\left(r_{1}, s_{1}\right) \odot\left(r_{2}, s_{2}\right)\right] \oplus\left[\left(r_{1}, s_{1}\right) \odot\left(r_{3}, s_{3}\right)\right],
\end{aligned}
$$

because for all $r \in R, s \in S,(r \cdot s, s \cdot s)=(r, s)$. Thus $\left(S^{-1} R, \oplus\right)$ is hypergroup. Also, it's clear that $\left(S^{-1} R, \odot\right)$ is associative and $(1,1)$ is as scalar identity and since $(R,+, \cdot)$ is commutative we obtain $\left(S^{-1} R, \oplus, \odot\right)$ is commutative. Hence $\left(S^{-1} R, \oplus, \odot\right)$ is the commutative hyperring with scalar identity.

Example 4.4. Let $(R,+,$.$) be a commutative ring with an identity 1$. Define a hyperoperation $x \circ y=\{x . y, 2 x . y, 3 x . y, \ldots\}$. Then $(R,+, \circ)$ is a commutative multiplicative hyperring which is not strongly distributive. Let $x \in R$ such that $x \notin \operatorname{nil}(R)$ and let $S=\left\{x^{n} \mid n \geq 0\right\}$. Then $\left(S^{-1} R, \oplus, \odot\right)$ is a commutative hyperring with an identity.

Theorem 4.5. Let $\left(R_{1},+,.\right)$ and $\left(R_{2},+^{\prime}, .^{\prime}\right)$ be two commutative multiplicative hyperrings with scalar identity 1 and $S$ be a MCS of $R_{1}$ and let $g: R_{1} \rightarrow R_{2}$ 
be a good homomorphism of multiplicative hyperrings such that $g\left(1_{R_{1}}\right)=1_{R_{2}}$. Then we have a good homomorphism $\hat{g}: S^{-1} R_{1} \rightarrow S^{-1} R_{2}$ defined by $\hat{g}(r, s)=$ $(g(r), g(s))$. Also, $\operatorname{ker}(\hat{g})=S^{-1} \operatorname{ker}(g)$.

Proof. Since $S$ is MCS in $R_{1}$, then we are able to see that $g(S)$ is MCS in $R_{2}$. Now, we need to show that $\hat{g}$ is well defined. Assume that $\left(r_{1}, s_{1}\right)=$ $\left(r_{2}, s_{2}\right)$, then there exists $A \subseteq S$ such that $\gamma_{1}^{*}\left(A \cdot r_{1} \cdot s_{2}\right)=\gamma_{1}^{*}\left(A \cdot r_{2} \cdot s_{1}\right)$. So, $g\left(\gamma_{1}^{*}\left(A \cdot r_{1} \cdot s_{2}\right)\right)=g\left(\gamma_{1}^{*}\left(A \cdot r_{2} \cdot s_{1}\right)\right)$ or $g\left(\gamma_{1}^{*}(A)\right) g\left(\gamma_{1}^{*}\left(r_{1}\right)\right) g\left(\gamma_{1}^{*}\left(s_{2}\right)\right)=$ $g\left(\gamma_{1}^{*}(A)\right) g\left(\gamma_{1}^{*}\left(r_{2}\right)\right) g\left(\gamma_{1}^{*}\left(s_{1}\right)\right)$. Since $\gamma_{2}^{*}(g(r))=g\left(\gamma_{1}^{*}(r)\right)$ for all $r \in R$, then we have

$$
\gamma_{2}^{*}(g(A)) \gamma_{2}^{*}\left(g\left(r_{1}\right)\right) \gamma_{2}^{*}\left(g\left(s_{2}\right)\right)=\gamma_{2}^{*}(g(A)) \gamma_{2}^{*}\left(g\left(r_{2}\right)\right) \gamma_{2}^{*}\left(g\left(s_{1}\right)\right)
$$

which implies that

$$
\gamma_{2}^{*}\left(g(A) \cdot^{\prime} g\left(r_{1}\right) \cdot^{\prime} g\left(s_{2}\right)\right)=\gamma_{2}^{*}\left(g(A) \cdot^{\prime} g\left(r_{2}\right) \cdot^{\prime} g\left(s_{1}\right)\right) .
$$

Since $g(A) \subseteq g(S)$, we have

$$
\left(g\left(r_{1}\right), g\left(s_{1}\right)\right)=\left(g\left(r_{2}\right), g\left(s_{2}\right)\right) \text { or } \hat{g}\left(r_{1}, s_{1}\right)=\hat{g}\left(r_{2}, s_{2}\right)
$$

Therefore $\hat{g}$ is well defined. Also $\hat{g}$ is a good homomorphism, because for all $\left(r_{1}, s_{1}\right),\left(r_{2}, s_{2}\right) \in S^{-1} R_{1}$ we have:

$$
\begin{aligned}
\hat{g}\left(\left(r_{1}, s_{1}\right) \oplus\left(r_{2}, s_{2}\right)\right) & =\hat{g}\left(r_{1} s_{2}+r_{2} s_{1}, s_{1} s_{2}\right) \\
& =\left(g\left(r_{1} \cdot s_{2}+r_{2} \cdot s_{1}\right), g\left(s_{1} s_{2}\right)\right) \\
& =\left(g\left(r_{1}\right) \cdot^{\prime} g\left(s_{2}\right)+^{\prime} g\left(r_{2}\right) \cdot^{\prime} g\left(s_{1}\right), g\left(s_{1}\right) \cdot^{\prime} g\left(s_{2}\right)\right) \\
& =\left(g\left(r_{1}\right), g\left(s_{1}\right)\right) \oplus^{\prime}\left(g\left(r_{2}\right), g\left(s_{2}\right)\right) \\
& =\hat{g}\left(r_{1}, s_{1}\right) \oplus^{\prime} \hat{g}\left(r_{2}, s_{2}\right) .
\end{aligned}
$$

Also we have

$$
\begin{aligned}
\hat{g}\left(\left(r_{1}, s_{1}\right) \odot\left(r_{2}, s_{2}\right)\right) & =\hat{g}\left(r_{1} \cdot r_{2}, s_{1} \cdot s_{2}\right) \\
& =\left(g\left(r_{1} \cdot r_{2}\right), g\left(s_{1} \cdot s_{2}\right)\right)=\left(g\left(r_{1}\right) \cdot^{\prime} g\left(r_{2}\right), g\left(s_{1}\right) \cdot^{\prime} g\left(s_{2}\right)\right) \\
& =\left(g\left(r_{1}\right), g\left(s_{1}\right)\right) \odot^{\prime}\left(g\left(r_{2}\right), g\left(s_{2}\right)\right) \\
& =\hat{g}\left(r_{1}, s_{1}\right) \odot^{\prime} \hat{g}\left(r_{2}, s_{2}\right) .
\end{aligned}
$$

Now for the last section, it's clear that $S^{-1} \operatorname{ker}(g) \subseteq \operatorname{ker}(\hat{g})$. Therefore we need to show $\operatorname{ker}(\hat{g}) \subseteq S^{-1} \operatorname{ker}(g)$. Assume that $(r, s) \in \operatorname{ker}(\hat{g})$, then $\hat{g}(r, s)=0$ or $(g(r), g(s))=0$. Thus $g(r)=0$ or $r \in \operatorname{ker}(g)$, i.e., $(r, s) \in S^{-1} \operatorname{ker}(g)$.

Theorem 4.6. Let $S$ be a MCS of a multiplicative hyperring $R$ with scalar identity 1. Then $S^{-1} R / \gamma_{1}^{*}=\left(\gamma_{2}^{*}(S)\right)^{-1}\left(R / \gamma_{2}^{*}\right)$.

Proof. It is enough to show that $\gamma_{1}^{*}(r, s)=\left(\gamma_{2}^{*}(r), \gamma_{2}^{*}(s)\right)$ for $r \in R, s \in S$. In order to achieve our claim, we have $\left(r^{\prime}, s^{\prime}\right) \in \gamma_{1}^{*}(r, s)$ if and only if there exist $\overline{z_{1}}, \overline{z_{2}}, \ldots, \overline{z_{n}}$ such that $\left\{(r, s),\left(r^{\prime}, s^{\prime}\right)\right\} \subseteq \prod_{i=1}^{n} \bar{z}_{i}$, where $\bar{z}_{i}=\left(r_{i}, s_{i}\right)$ for $r_{i} \in R, s_{i} \in S$. It holds, if and only if $\left\{(r, s),\left(r^{\prime}, s^{\prime}\right)\right\} \subseteq\left(\prod_{i=1}^{n} r_{i}, \prod_{i=1}^{n} s_{i}\right)$ 
if and only if $\left\{r, r^{\prime}\right\} \subseteq \prod_{i=1}^{n} r_{i}$ and $\left\{s, s^{\prime}\right\} \subseteq \prod_{i=1}^{n} s_{i}$ if and only if $r^{\prime} \in$ $\gamma_{2}^{*}(r), s^{\prime} \in \gamma_{2}^{*}(s)$ if and only if $\left(r^{\prime}, s^{\prime}\right) \in\left(\gamma_{2}^{*}(r), \gamma_{2}^{*}(s)\right)$. Hence $S^{-1} R / \gamma_{1}^{*}=$ $\left(\gamma_{2}^{*}(S)\right)^{-1}\left(R / \gamma_{2}^{*}\right)$.

It is easy to check that $\phi: R \rightarrow S^{-1} R$ defined by $\phi(r)=(r, 1)$ is a good homomorphism. Now, let $I$ be a hyperideal of multiplicative hyperring $R$, then we can define that $S^{-1} I=\{(i, s) \mid i \in I, s \in S\}$, which is a hyperideal of $S^{-1} R$.

Remark 4.7. If $(r, s) \in S^{-1} I$ we don't have necessarily $r \in I$, because maybe $(r, s)=\left(r^{\prime}, s\right)$ with $r^{\prime} \in I, r \notin I$.

Theorem 4.8. Let $S$ be a MCS of a multiplicative hyperring $R$ with scalar identity 1 and $I$ and $J$ be two hyperideals in $R$, then we have the following statements:

(i) $S^{-1}(I+J)=S^{-1} I+S^{-1} J$

(ii) $S^{-1}(I \cdot J)=\left(S^{-1} I\right)\left(S^{-1} J\right)$

(iii) $S^{-1}(I \cap J)=S^{-1} I \cap S^{-1} J$.

Proof. It is straightforward.

Theorem 4.9. Let $I$ be a $\mathcal{C}$-ideal and $S$ be a MCS of multiplicative hyperring $R$ with scalar identity 1 . Then $I \cap S \neq \emptyset$ if and only if $S^{-1} I=S^{-1} R$.

Proof. $(\Rightarrow)$ Assume that $x \in I \cap S$, then $(1,1)=(x, x) \in S^{-1} I$. So $(1,1) \odot$ $(r, s) \in S^{-1} I$ for all $(r, s) \in S^{-1} R$. Therefore $(r, s) \in S^{-1} I$. Hence $S^{-1} I=$ $S^{-1} R$.

$(\Leftarrow)$ Consider the inclusion homomorphism $\phi: R \rightarrow S^{-1} R$, then $\phi(1)=(1,1)$. Since $\phi(1) \in S^{-1} R$ and $S^{-1} I=S^{-1} R$, then $\phi(1) \in S^{-1} I$. So, there exist $i \in I, s \in S$ such that $(1,1)=\phi(1)=(i, s)$. Therefore, there exists $A \subseteq S$ such that $\gamma^{*}(A \cdot i)=\gamma^{*}(A \cdot s)$. Since $A \cdot i \subseteq I$ and $A \cdot i \subseteq \gamma^{*}(A \cdot i)$, then $I \cap \gamma^{*}(A \cdot i) \neq \emptyset$ and on the other hands $I$ is $\mathrm{C}$-ideal, then we have $A \cdot s \subseteq \gamma^{*}(A \cdot s)=\gamma^{*}(A \cdot i) \subseteq I$. Hence $I \cap S \neq \emptyset$.

Corollary 4.10. Let $I$ and $J$ be two hyperideals and $S$ be a MCS of multiplicative hyperring $R$ with scalar identity 1 such that $I+J$ is $\mathcal{C}$-ideal. Then $I$ and $J$ are coprime in $R$ if and only if $S^{-1} I$ and $S^{-1} J$ are coprime in $S^{-1} R$.

Proof. $(\Rightarrow)$ Assume that $I$ and $J$ are coprime, then $I+J=R$, and by Theorem 4.8 $(i)$ we have $S^{-1} R=S^{-1} I+S^{-1} J$, i.e., $S^{-1} I$ and $S^{-1} J$ are coprime in $S^{-1} R$.

$(\Leftarrow)$ Since $S^{-1} I$ and $S^{-1} J$ are coprime in $S^{-1} R$, we have $S^{-1} R=S^{-1} I+$ $S^{-1} J=S^{-1}(I+J)$. Thus we observe that $(I+J) \cap S \neq \emptyset$ and since $I+J$ is C-ideal we have $S \subseteq I+J$ and since $1 \in S$, we have $I+J=R$. 
Theorem 4.11. If $I$ is a nilpotent hyperideal of multiplicative hyperring $R$ with scalar identity 1 , and $S^{-1} I$ is a hyperideal of $S^{-1} R$, then $S^{-1} I$ is nilpotent.

Proof. We need to show that for any sequence $\left(r_{1}, s_{1}\right),\left(r_{2}, s_{2}\right), \ldots$ in $S^{-1} I$, there are $a_{i}^{\prime} \in I$ and $s_{i}^{\prime} \in S$ such that for each $n$ we have

$$
\left(r_{n}, s_{n}\right) \odot\left(r_{n-1}, s_{n-1}\right) \odot \ldots \odot\left(r_{1}, s_{1}\right)=\left(r_{n}^{\prime} \ldots r_{1}^{\prime}, s^{\prime} n\right) .
$$

In order to achieve our claim, we use induction. Assume that we have found them for $i \leq n-1$, then $\left(r_{n-1}, s_{n-1}\right) \odot \ldots \odot\left(r_{1}, s_{1}\right)=\left(r_{n-1}^{\prime} \cdot \ldots \cdot r_{1}^{\prime}, s_{n-1}^{\prime}\right)$. By our assumption $A=\left(r_{n}, s_{n}\right) \odot\left(1, s_{n-1}^{\prime}\right) \subseteq S^{-1} I$, then for suitable $r_{n}^{\prime} \in I$ and $s_{n}^{\prime} \in S$, we have $A=\left(r_{n}^{\prime}, s_{n}^{\prime}\right)$. Therefore,

$$
\begin{aligned}
\left(r_{n}, s_{n}\right) \odot & \left(r_{n-1}, s_{n-1}\right) \odot \ldots \odot\left(r_{1}, s_{1}\right)=\left(r_{n}, s_{n}\right) \odot\left(r_{n-1}^{\prime} \cdot \ldots \cdot r_{1}^{\prime}, s_{n-1}^{\prime}\right) \\
& =A \odot\left(a_{n-1}^{\prime} \cdot \ldots \cdot a_{1}^{\prime}, 1\right)=\left(a_{n}^{\prime}, s_{n}^{\prime}\right) \odot\left(a_{n-1}^{\prime} \cdot \ldots \cdot a_{1}^{\prime}, 1\right) \\
& =\left(a_{n}^{\prime} \cdot a_{n-1}^{\prime} \cdot \ldots \cdot a_{1}^{\prime}, s_{n}^{\prime}\right),
\end{aligned}
$$

hence our claim holds. Now our claim follow that, if there exists $n$ such that $0 \in I^{n}$ then $0 \in\left(S^{-1} I\right)^{n}$.

Theorem 4.12. Let $R$ be a commutative multiplicative hyperring with scalar identity 1 and $S$ be a MCS. Then we have the following statements:

(i) Every hyperideal in $S^{-1} R$ is an extension hyperideal.

(ii) If $I$ is an $\mathcal{C}$-ideal of $R$, then $I^{e c}=\cup_{s \in S}(I: s)$.

(iii) Let $I$ be a $\mathcal{C}$-ideal of $R$ and $\mathfrak{D}=\left\{J \mid J^{e c}=J\right\}$. Then $I \in \mathfrak{D}$ if and only if no element of $S$ is a zero divisor in $R / I$.

Proof. ( $i$ ) Let $J$ be a hyperideal in $R$ and let $(r, s) \in J$. Since $(r, 1)=(r, s) \odot$ $(s, 1)$, then $(r, 1) \in J$, hence $r \in J^{c}$ and therefore $(r, s) \in J^{c e}$. Since $J^{c e} \subset J$, then $J=J^{c e}$.

(ii) For all $r \in R, r \in I^{e c}=\left(I^{e}\right)^{c}=\left(S^{-1} I\right)^{c}$ if and only if $(r, 1)=(i, s)$ for some $i \in I, s \in S$ if and only if there exists $A \subseteq S$ such that $\gamma^{*}(A \cdot r \cdot i)=$ $\gamma^{*}(A \cdot s)$ if and only if $A \cdot s \cdot i \subseteq I$ (since $\gamma^{*}(A \cdot s)=\gamma^{*}(I)$ and $0 \in \gamma^{*}(I)$ and since $I$ is $\mathrm{C}$-ideal, therefore $\left.A \cdot s \cdot i \subseteq \gamma^{*}(A \cdot s \cdot i) \subseteq I\right)$. Hence, it holds if and only if $r \in \cup_{s \in S}(I: s)$.

(iii) For all hyperideal $I$ of $R, I \in \mathfrak{D}$ if and only if $I^{e c}=I$ if and only if for some $s \in S$ and $r \in R$ such that $s \cdot r \subseteq I$ implies $r \in I$. Hence, it holds if and only if no $s \in S$ is a zero divisor in $R / I$, because if there exists $s \in S$ such that $I \subseteq s \cdot(r+I)=s \cdot r+I$, then $s \cdot r \cap I \neq \emptyset$. Since $I$ is -ideal then $s \cdot r \subseteq I$, hence $r \in I$ and it is contradiction. 
Theorem 4.13. Let $R$ be a commutative multiplicative hyperring with scalar identity 1 and $S$ be a MCS and every prime hyperideal of $R$ is $\mathcal{C}$-ideal. Then the prime hyperideals of $S^{-1} R$ are in one to one correspondence with the prime hyperideals of $R$ which don't meet $S$.

Proof. Assume that $P$ is a prime hyperideal in $S^{-1} R$, then $P^{c}$ is a prime hyperideal in $R$. Conversely, let $Q$ be a prime hyperideal in $R$. Then $\frac{R}{Q}$ is a hyperdomain. Now define $\phi: S^{-1} R \rightarrow \bar{S}^{-1}\left(\frac{R}{Q}\right)$ by $\phi(r, s)=(r+Q, s+Q)$. It is clear that $\phi$ is well define and good homomorphism and onto. Also we have for all $(r, s) \in S^{-1} R$ such that $\phi(r, s)=0_{\bar{S}^{-1}\left(\frac{R}{Q}\right)}$, then $(r+Q, s+Q)=(Q, s+Q)$. Therefore there exists $\bar{A} \subseteq \bar{S}$ such that $\gamma^{*}(A \cdot r \cdot s+Q)=\gamma^{*}(Q)$. Then there exists

$$
\bar{z}_{1}, \bar{z}_{2}, \ldots, \bar{z}_{n} \text { in } \frac{R}{Q}
$$

such that

$$
A \cdot r \cdot s+Q, Q \subseteq \prod_{i=1}^{n} \bar{z}_{i}=\prod_{i=1}^{n} z_{i}+Q
$$

i.e.,

$$
A \cdot r \cdot s+Q \subseteq \prod_{i=1}^{n} z_{i}+Q \text { and } Q \subseteq \prod_{i=1}^{n} z_{i}+Q
$$

Thus $Q \cap \prod_{i=1}^{n} z_{i} \neq \emptyset$ and $\left(A \cdot r \cdot s-\prod_{i=1}^{n} z_{i}\right) \cap Q \neq \emptyset$. Since $Q$ is a $\mathcal{C}$ ideal, then $A \cdot r \cdot s \subseteq Q$ and since $S \cap Q=\emptyset$ and $Q$ is prime, then $r \in Q$. Hence $\operatorname{ker}(\phi)=S^{-1} Q$, so we have $\frac{S^{-1} R}{S^{-1} Q} \cong \bar{S}^{-1}\left(\frac{R}{Q}\right)$, which is either 0 or else is contained in the hyperfield of fractions of $\frac{R}{Q}$ and is then a hyperdomain and so $S^{-1} Q$ is either prime or is the unit hyperideal and the latter occurs if and only if $Q$ meets $S$, by Theorem $4.11(i)$.

\section{Acknowledgements.}

The first author has been partially supported by the "Research Center in Algebraic Hyperstructures and Fuzzy Mathematics, University of Mazandaran, Babolsar, Iran" and "Algebraic Hyperstructure Excellence, Tarbiat Modares University, Tehran, Iran". The third author has been supported within the project "VÝZKUMFVT (DZRO K-217)" supported by the Ministry of Defence, Czech Republic.

\section{References}

[1] R. Ameri, M. Norouzi, New fundamental relation of hyperrings, European J. Combin., 34 (2013) 884-891. 
[2] R. Ameri, M. Norouzi, Prime and primary hyperideals in Krasner $(m, n)$ - hyperrings, European J. Combin., 34 (2013) 379-390.

[3] R. Ameri, S. Hoskova-Mayerova, M. Amiri-Bideshki, A. Borumand Saeid, Prime filters of hyperlattices. An. Stiint. Univ. "Ovidius" Constanta Ser. Mat., 24(2) (2016) 15-26.

[4] A. Asokkumar, M. Velrajan, Characterizations of regular hyperrings, Ital. J. Pure Appl. Math., 22 (2007) 115-124.

[5] A. Asokkumar, M. Velrajan, Hyperring of matrices over a regular hyperring, Ital. J. of Pure Appl. Math., 23 (2008) 13-120.

[6] P. Corsini, Prolegomena of hypergroup theory, Second ed., Aviani Editore, 1993.

[7] P. Corsini, V. Leoreanu, Applications of hyperstructures theory, Adv. Math., Kluwer Academic Publishers, 2003.

[8] J. Chvalina, J., Š. Křehlík, M. Novák, Cartesian composition and the problem of generalizing the MAC condition to quasi- multiautomata, $A n$. Stiint. Univ. "Ovidius" Constanta Ser. Mat., 24(3) (2016) 79-100.

[9] I. Cristea, S. Jancic-Rasovic, Compositions Hyperrings, An. Stiint. Univ. "Ovidius" Constanta Ser. Mat., 21(2) (2013) 81-94.

[10] I. Cristea, Regularity of Intuitionistic Fuzzy Relations on Hypergroupoids, An. Stiint. Univ. "Ovidius" Constanta Ser. Mat., 22(1) (2014) 105-119.

[11] U. Dasgupta, Prime and primary hyperideals of a multiplicative hyperring, An. Stiint. Univ. Al. I. Cuza Iasi.Mat., 58(1) (2012) 19-36.

[12] B. Davvaz, S. Mirvakili, On $\alpha$-relation and transitive condition of $\alpha$, Commun. Algebra, 36(5) (2008) 1695-1703.

[13] B. Davvaz, T. Vougiouklis, Commutative rings obtained from hyperrings ( $H_{v}$-rings) with $\alpha^{*}$-relations, Comm. Algebra, 35 (2007) 3307-3320.

[14] B. Davvaz, V. Leoreanu-Fotea, Hyperring theory and applications, International Academic Press, USA, 2007.

[15] M. De Salvo, G. Lo Faro, On the $n^{*}$-complete hypergroups, Discrete Math. 208/209 (1990) 177-188.

[16] D. Freni, A new characterization of the derived hypergroup via strongly regular equivalences, Comm. Algebra, 30(8) (2002) 3977-3989. 
[17] D. Freni, Strongly transitive geometric spaces: Applications to hypergroups and semigroups theory, Comm. Algebra, 32 (2004) 969-988.

[18] M. Koskas, Groupoids, demi-groupes et hypergroupes, J. Math. Pures Appl., 49 (1970) 155-192.

[19] M. Krasner, A class of hyperrings and hyperfields, Int. J. Math. Math. Sci. 2 (1983) 307-312.

[20] F. Marty, Sur une generalization de la notion de groupe, In: 8iem Congres des Mathematiciens Scandinaves, Stockholm, (1934) 45-49.

[21] C. G. Massouros, On the theory of hyperrings and hyperfields, Algebra i Logika, 24 (1985) 728-742.

[22] J. Mittas, Hypergroups canoniques, Math. Balkanica, 2 (1972) 165-179.

[23] J. Mittas, Sur les hyperanneaux et les hypercorps, Math. Balkanica, 3 (1973), 368-382.

[24] A. Nakassis, Recent Result in hyperring and Hyperfield Theory, Int. J. Math. Math. Sci, 11(2) (1988) 209-220.

[25] M. Novák, n-ary hyperstructures constructed from binary quasi-orderer semigroups, An. Stiint. Univ. "Ovidius" Constanta Ser. Mat., 22(3) (2014) 147-168.

[26] D. M. Olson and V.K. Ward,A note on multiplicative hyperrings, Italian J. Pure Appl. Math., 1 (1997) 77-84.

[27] C. Pelea, Hyperrings and $\alpha^{*}$-relations. A general approach, J. Algebra, 383, 1 (2013) 104-128.

[28] C. Pelea, I. Purdea and L. Stanca Factor multialgebras, universal algebras and fuzzy sets, Carpathian J. Math. 31(1) (2015), 111-118.

[29] R. Procesi-Ciampi, R. Rota, The hyperring spectrum, Riv. Mat. Pura Appl., 1 (1987) 71-80.

[30] R. Procesi, R. Rota, On some classes of hyperstructures, Combinatorics Discrete Math., 208/209 (1999) 485-497.

[31] A. Rahnamai Barghi, A class of hyperrings, J. Discrete Math. Sci. Cryptogr., 6 (2003) 227-233.

[32] R. Rota, Strongly distributive multiplicative hyperrings, J. Geom., 39 (1990) 130-138. 
[33] R. Rota, Sugli iperanelli moltiplicativi, Rend. Di Mat., Series VII (4), 2 (1982) 711-724.

[34] R. Rota, Congruenze sugli iperanelli moltiplicativi, Rend. Di Mat., Series VII (1), 3 (1983) 17-31.

[35] R. Rota, Sulla categoria degli iperanelli moltiplicativi, Rend. Di Mat., Series VII (1), 4 (1984) 75-84.

[36] S. Spartalis, T. Vougiouklis, The fundamental relations on $H_{v}$-rings, Math. Pure Appl., 13 (1994) 7-20.

[37] S. Spartalis, A class of hyperrings, Riv. Mat. Pura Appl.,4 (1989), 55-64.

[38] S. Spartalis, (H;R)-hyperrings, Algebraic hyperstructures and applications (Xanthi, 1990), 187-195, World Sci. Publ., Teaneck, NJ, 1991.

[39] D. Stratigopoulos, Hyperanneaux non commutatifs: Le radical d'un hyperanneau, somme sous-directe des hyperanneaux artiniens et theorie des elements idempotents, C.R. Acad. Sci. Paris, 269 (1969), 627-629.

[40] T. Vougiouklis, Hyperstructures and Their Representations, Hadronic Press, Inc., Palm Harber, USA, 1994.

[41] T. Vougiouklis, The fundamental relation in hyperrings, The general hyperfield, In: Proc. Fourth Int. Congress on Algebraic Hyperstructures and Applications, AHA, 1990, World Scientific, 1991, 203-211.

[42] M. M. Zahedi, R. Ameri, On the prime, primary and maximal subhypermodules, Ital. J. Pure Appl. Math., 5 (1999) 61-80.

Reza AMERI,

School of Mathematics, Statistics and Computer Science,

College of Sciences, University of Tehran,

P.O. Box 14155-6455, Teheran, Iran.

Email: rameri@ut.ac.ir

Ali KORDI,

School of Mathematics, Statistics and Computer Science,

College of Sciences, University of Tehran,

P.O. Box 14155-6455, Teheran, Iran.

Email: ali.kordi@gmail.com

Sarka HOSKOVA-MAYEROVA

Department of Mathematics and Physics,

University of Defence, Brno

Kounicova 65, 66210 Brno, Czech Republic.

Email: sarka.mayerova@unob.cz 